\title{
CAPACIDAD PREDICTIVA DE LA ESCALA DE PROCRASTINACIÓN ACADÉMICA EN ESTUDIANTES UNIVERSITARIOS
}

\section{PREDICTIVE CAPACITY OF THE ACADEMIC PROCRASTINATION SCALE IN UNIVERSITY STUDENTS}

Edward Faustino Loayza-Maturrano ${ }^{1}$

iD https://orcid.org/0000-0002-1359-8414

edwloma@lamolina.edu.pe

Aceptado:20/10/2021

Publicado online:30/11/2021

\begin{abstract}
RESUMEN
El objetivo de esta investigación es determinar la capacidad predictiva de la escala de procrastinación académica en estudiantes universitarios respecto de su rendimiento académico. La metodología empleada en este estudio es cuantitativa de tipo correlacional-transversal. Se aplicó el cuestionario de procrastinación académica de Solomon y Rothblum (1984) a 74 estudiantes distribuidos en tres cursos (lenguaje y comunicación, redacción técnica y comprensión textual) de la Universidad Nacional Agraria La Molina. Los resultados evidenciaron que el 31,9\% de estudiantes tienen procrastinación académica entre los baremos "siempre" o "casi siempre". Además, el $45,15 \%$ de los estudiantes afirmó que la procrastinación académica frecuente o moderada les había causado problemas académicos. Hubo una correlación inversa significativa entre la procrastinación académica y el rendimiento académico de los estudiantes universitarios $(p<0.01)$. También, los estudiantes varones mostraron más altos niveles de dilación que las mujeres ( $p$ $<0,01)$. En este estudio se concluye que los estudiantes que evidencian al tos niveles de procrastinación académica, muestran un rendimiento académico inferior o abandonan el curso.
\end{abstract}

Palabras clave: Dilación académica; causas de procrastinación; escala de procrastinación académica; rendimiento académico; estudiantes universitarios.

\begin{abstract}
The objective of this research is to determine the predictive capacity of the academic procrastination scale in university students regarding their academic performance. The methodology used in this study is quantitative, correlational-
\end{abstract}

\footnotetext{
1 Universidad Nacional Agraria La Molina, Facultad de Economía y Planificación, Departamento de Ciencias Humanas y Educación. Maestro en Ciencias dela Educación.
} 
cross-sectional. The Solomon and Rothblum (1984) academic procrastination questionnaire was applied to 74 students distributed in three courses (language and communication, technical writing and textual comprehension) of the Universidad Nacional Agraria La Molina. The results showed that $31.9 \%$ of students have academic procrastination between the scales "always" or "almost always". Furthermore, $45.15 \%$ of the students stated that frequent or moderate academic procrastination had caused them academic problems. There was a significant inverse correlation between academic procrastination and academic performance of university students ( $p<0.01$ ). Also, male students showed higher levels of procrastination than females $(p<0.01)$. This study concludes that students who show high levels of academic procrastination show lower academic performance or drop out of the course.

Keywords: Predictor variables; student behavior; college students; student responsibility; time management.

\section{INTRODUCCIÓN}

En los últimos años, la mejora del rendimiento académico de los estudiantes universitarios ha sido uno de los temas más importantes en la educación superior y uno de los principales desafíos que las instituciones de formación universitaria pretenden alcanzar, por lo que surge la necesidad de encontrar estrategias para elevar el rendimiento académico de los estudiantes de la educación terciaria. Así se han realizado muchos estudios sobre los factores que inciden en el aumento del rendimiento de los estudiantes (González-Pienda et al. 2003; Gómez, Martínez \& Oviedo, 2011; Vargas \& Montero, 2016; Hernández et al., 2021); sin embargo, se han realizado en menor proporción estudios sobre factores que previenen el bajo rendimiento (Pérez-Mato \& Sierra-Salcedo, 2010; Erazo, 2011; Medina, Ferreira \& Marzo, 2018). De hecho, investigar los factores que previenen el bajo rendimiento es tan importante como investigar los factores que promueven el rendimiento académico.

En este sentido, la procrastinación se considera un fenómeno prevalente que interfiere con el desempeño académico y las actividades cotidianas de los individuos (Quant \& Sánchez, 2012). A pesar de que algunas de las conductas dilatorias pueden considerarse conductas intencionadas, la postergación académica se considera ilógica y a menudo destructiva dado que muchos estudiantes lo practican, retrasando sus tareas académicas sin una razón lógica, pese a ser conscientes de los resultados negativos que le ocasionan (Díaz-Morales, 2019).

Además, con el avance de la tecnología, cada vez hay más opciones de ocio y entretenimiento. Muchas veces, para esto, una persona ni siquiera tiene que salirde su propia casa, lo que genera muchas formas de distraerse de realizar una actividad importante para él, ya sea hacer el trabajo o las tareas del hogar, el autodesarrollo, las actividades educativas. La procrastinación académica, que se manifiesta en el aplazamiento de las tareas de aprendizaje, es un problema grave para los estudiantes. Por ejemplo, los estudiantes que recién ingresan a la universidad obtienen mucha libertad personal. Se supone que deben organizar su propio tiempo para aprender, sin embargo, a causa de la falta de habilidades de autorregulación, los estudiantes caen en la procrastinación. Primero, pos ponen hacer sus deberes por un día, luego por dos. En última instancia, el trabajo permanece inacabado hasta la fecha límite, por lo que 
el estudiante debe completar todo en condiciones severamente restringidas. Como resultado, no solo se ve afectada la calidad del trabajo y la calificación obtenida, sino también el propio discente, porque tanto el proceso de procrastinación como el trabajo en las asignaciones bajo plazos ajustados van acompañados de una fuerte tensión interna, aumento de la ansiedad, la ira y la depresión. Consecuentemente, el rendimiento académico general disminuye, incluso puede llegar a la expulsión.

En este sentido, la procrastinación en la personalidad puede definirse como un retraso voluntario en actividades importantes y planificadas a pesar de la expectativa de posibles consecuencias negativas que superan las consecuencias positivas del retraso. En consecuencia, se entiende por procrastinación a la acción de ignorar, retrasar, posponer, prolongar y diferir la realización de una actividad o tarea (Natividad, 2014). De este modo, se entiende que es en el entorno de aprendizaje donde surgen con mayor frecuencia las condiciones para la aparición de la procrastinación, lo que conduce a consecuencias negativas no solo en la universidad, sino también en el futuro y en la construcción de una carrera profesional.

Por ello, como una forma de comportamiento de procrastinación, la procrastinación académica es una tendencia irracional a través de la cual uno retrasa el comienzo o el final de las tareas o asignaciones del curso. Este comportamiento es muy común entre los estudiantes, especialmente en lo que se refiere a trabajos encargados para los que existe un plazo predeterminado (Schouwenburg, 2004). Cabe mencionar que la vida de los estudiantes implica plazos frecuentes para la inscripción, selección de cursos, envío de trabajos, artículos, etc. Una forma común de procrastinación de los estudiantes es posponer la redacción de trabajos o dilatar el estudiar para los exámenes (Valenzuela et al., 2020).

Según Valeria De Palo et al. (2017) la procrastinación es uno de los factores más importantes que causan dificultades de aprendizaje; no obstante, el principal enemigo de este fenómeno es la presencia de un objetivo claro y constante en la vida de la persona, al cual intentará esforzarse independientemente de los acontecimientos que le rodean. Asimismo, Ann Krispenz et al. (2019) ha demostrado que los grupos con altos y bajos niveles de procrastinación se caracterizan por diferentes tipos de motivación. Así, los procrastinadores demuestran una motivación controlada externamente más pronunciada, mientras que los no procrastinadores están dominados por una motivación intrínseca autónoma. Los investigadores concluyen que la dilación puede tener un efecto destructivo en la autodeterminación personal, social y profesional de un individuo. Al mismo tiempo, la procrastinación en la estrategia de vida general y la esfera profesional tiene el efecto más destructivo a una edad temprana Zacks \& Hen, 2018).

La procrastinación, como comportamiento irracional y destructivo, puede llevar a la reducción de la confianza en uno mismo y la somnolencia, y aumentan los comportamientos poco saludables, tabaquismo, bebida, trastornos gastrointestinales, insomnio, ansiedad y depresión, reduciendo así la calidad de vida (Steel \& Gröpel, 2008; Krispenz et al., 2019). Los resultados de algunos estudios revelaron que los altos niveles de procrastinación impedían a los estudiantes organizar sus metas académicas y alcanzarlas. El rendimiento académico está en mayor riesgo entre los estudiantes que postergan las cosas, antes que entre quienes cumplen las actividades en los plazos previstos y no muestran dilación. Varios estudios han concluido que la procrastinación afecta negativamente el aprendizaje, la actitud hacia los cursos y el rendimiento académico (Visseret al, 2018). Además, según algunos estudios, existe 
una relación negativa entre la dilación y las creencias racionales sobre el estudio, la satisfacción con la vida académica, la autoeficacia y el uso de estrategias de aprendizaje metacognitivo (De Palo et al., 2017).

En este sentido, Ann Krispenz et al. (2019) señala que una disminución en la motivación académica y profesional entre los estudiantes se manifiesta principalmente en una situación de cambio de modelo de enseñanza, en particular, durante la transición de actividades educativas a prácticas en vísperas y en las etapas iniciales de la formación práctica, así como en la situación de la necesidad de optar por un rumbo posterior a la actividad profesional. Así, un estado de personalidad negativo, por ejemplo, la ansiedad antes de la prueba, también puede afectar negativamente a la esfera motivacional de la personalidad, provocando una tendencia a la evitación y dando lugar a una procrastinación académica en la actividad (Karatas, 2015; De Palo et al., 2019).

Los resultados del estudio de Carola Grunschel et al. (2016) sobre estudiantes universitarios revelaron que los procrastinadores tenían puntajes más bajos que otros estudiantes. Un hallazgo principal de esta investigación deriva en la conexión de la procrastinación y la atención plena. Primero, una mayor atención plena conduce a bajas tasas de procrastinación. Esto significa que, si la gente está atenta, ahorrará menos para más adelante. En segundo lugar, por el contrario, el estado de alerta bajo conduce a una mayor procrastinación. La falta de atención está estrechamente relacionada con las emociones negativas, que también es la causa de la procrastinación. En fin, aunque los resultados negativos de este hábito en la vida diaria de uno pueden no ser considerables, las consecuencias de su prevalencia entre los estudiantes universitarios que son el asumir responsabilidades importantes en el futuro como profesionales, puede ser irreparable. Por tanto, es fundamental identificar la correlación entre la postergación académica y el rendimiento académico; es decir, en qué proporción existe una relación directa entre la procrastinación académica y el éxito académico del estudiante (Hen \& Goroshit, 2020).

De este modo, Murat Balkis (2013) explica que la procrastinación puede afectar el rendimiento académico de diferentes maneras: 1 ) Los procrastinadores sienten que no están lo suficientemente preparados para completar la tarea $y$, por lo tanto, la evitan hasta que tienen suficiente tiempo; 2) No invierten el tiempo y el esfuerzo necesarios para un buen trabajo debido a la subestimación del tiempo; 3) los procrastinadores enfrentan obstáculos imprevistos que surgen más cerca del inicio de los plazos.

Igualmente, necesario es determinar las principales causas que generan la procrastinación académica en los universitarios, por lo que el empleo de un instrumento que permita observar tanto las dimensiones de la procrastinación académica como la prevalencia de las principales causas de la dilación es esencial para lograr el análisis de este problema (Balkis \& Duru, 2019). En tal sentido, se halló el cuestionario de procrastinación académica de Solomon y Rothblum (1984) que permite observar todos estos aspectos de la procrastinación académica y, asimismo, posibilita establecer una correlación con los resultados académicos de los universitarios. Así, el objetivo principal de este estudio es determinar la capacidad predictiva de la procrastinación académica en función de los niveles de rendimiento académico de los estudiantes universitarios. Para este fin se analizaron las relaciones de la capacidad predictiva de los constructos del cuestionario de procrastinación académica de Solomon y Rothblum sobre el rendimiento académico. Esta variable de éxito académico se 
operacionaliza a partir de los promedios finales obtenidos por los estudiantes en los cursos desarrollados.

\section{METODOLOGÍA}

Diseño. La investigación desarrolla una metodología de tipo cuantitativa de diseño correlacional-transversal, debido a que se describe cómo la escala adaptada de procrastinación académica demuestra capacidad predictiva respecto de los niveles de rendimiento académico en los estudiantes universitarios (Loayza, 2020). El estudio realiza el análisis en un solo momento sin considerar evolución o tendencia por lo que permitirá describir el fenómeno sin considerar la variable temporal ni las variables extrañas que surjan en una dimensión longitudinal (Loayza, 2006; Namakforoosh, 2009). De este modo, se planteó la hipótesis general de investigación $\left(\mathrm{H}_{1}\right)$ : La aplicación del cuestionario de procrastinación académica adaptado de Solomon \& Rothblum (1984) permite predecir significativamente el nivel de rendimiento académico de los estudiantes universitarios. Asimismo, el diseño transversal que admite alcanzar la indagación en un solo momento (un solo tiempo determinado) se grafica en la Figura 1 , donde $M$ es muestra (74 estudiantes agrupados por conglomerados), $\mathrm{V}_{1}$ es la variable procrastinación académica, $\mathrm{V}_{2}$ es la variable rendimiento académico y $r$ indica la relación entre las variables.

Figura 1. Esquema del diseño correlacional

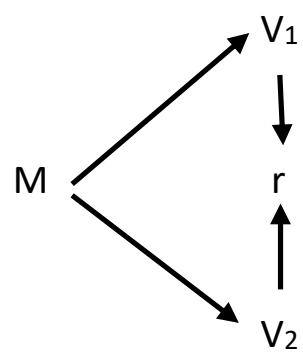

Participantes. El cuestionario se aplicó a 74 estudiantes distribuidos en tres cursos (lenguaje y comunicación, redacción técnica y comprensión textual) que se imparten en la Universidad Nacional Agraria La Molina. La selección de los participantes fue de acuerdo a las inscripciones al azar de estudiantes a los cursos focalizados en la institución universitaria de estudio. Se aprovechó la disponibilidad del acceso remoto a una encuesta en la plataforma Google forms con el propósito de facilitar la resolución del cuestionario.

Instrumento. Se empleó el cuestionario de procrastinación académica de Solomon \& Rothblum (1984), cuyo repertorio léxico fue adaptado a las características discursivas del contexto académico peruano. La elección del instrumento se debió a dos razones principales: $1^{\circ}$. La confiabilidad del instrumento en distintas poblaciones y en distintas sociedades como la occidental y la oriental. Así se ha aplicado en contextos como el norteamericano y el musulmán. $2^{\circ}$. Un cuestionario que analiza de forma más abarcadora el fenómeno de la procrastinación académica; porque las dimensiones, los criterios e ítems que considera en su diseño, abordan en un mismo instrumento las características ylas causas de la procrastinación académica. La validez y fiabilidad del cuestionario adaptado fueron confirmadas por la aplicación preliminar piloto del mismo cuestionario a otros dos grupos de 12 estudiantes del curso de Lengua. Según la prueba de confiabilidad del instrumento se obtuvo una fiabilidad Alfa de Cronbach de 0,916. 
En esta investigación se analizan las dos dimensiones de la procrastinación académica: a) El comportamiento de procrastinación de estudiantes universitarios y b) Las causas principales de procrastinación académica. En la primera dimensión se explora el comportamiento de procrastinación de los estudiantes universitarios en seis criterios: (1) elaboración de un trabajo final de curso, (2) estudiar para los exámenes, (3) cumplimiento de la asignación de lectura semanales, (4) ejecución de actividades administrativas académicas, (5) cumplimiento de acciones de asistencia y (6) ejecución de actividades académicas en general. Para cada criterio, se designaron tres preguntas, con un total de 18 preguntas. Estos ítems se puntuaron en una escala Likert de cinco puntos desde 1 (nunca / no quiero disminuirlo) a 5 (siempre / definitivamente quiero disminuirlo). Así, la cantidad de procrastinación académica y los problemas que causó al individuo se calificaron de 18 a 90. El puntaje total varió de 12 a 60 . Según el sistema de calificación, cuanto mayor sea la calificación, mayor será la procrastinación académica.

En la segunda dimensión, se explora las causas principales de procrastinación académica en trece criterios: (1) ansiedad por la evaluación, (2) perfeccionismo, (3) dificultades para tomar decisiones, (4) dependencia y búsqueda de ayuda, (5) aversión a la tarea y baja tolerancia a la frustración, (6) falta de confianza en sí mismo, (7) falta de aprobación, (8) pereza, (9) miedo al éxito, (10) tendencia a sentirse abrumado y administrar mal el tiempo, (11) rebelión contra el control, (12) tomar riesgos, (13) influencia de los compañeros Para cada criterio, se designaron dos preguntas, con un total de 26 preguntas. Estos ítems se puntuaron en una escala Likert de tres puntos desde 1 (no, de ningún modo refleja por qué yo postergo la tarea) a 3 (sí, definitivamente refleja por qué yo postergo la tarea). Así, las causas de procrastinación académica se calificaron de 26 a 78. El puntaje total según el sistema de calificación, cuanto mayor sea la calificación del criterio, éste se va definiendo como una de las causas principales de la procrastinación académica. Finalmente, el instrumento empleado para este estudio, completo y adaptado con sus dimensiones y criterios se puede revisar en https://osf.io/zxs3y/.

Procedimiento. El instrumento se aplicó individualmente con una duración de 30 minutos. La recogida de datos se efectuó en el mes de octubre de 2020. Asimismo, en un apartado preliminar del instrumento se explicó la naturaleza e importancia del estudio y se indicó el carácter anónimo y la condición voluntaria de la participación. El análisis de los datos se realizó aplicando estadísticas descriptivas e inferenciales (coeficiente de correlación de Pearson, Prueba t-student independiente y el análisis de varianza ANOVA unidireccional) a través del programa IBM-SPSS versión 26. La correlación se efectuó entre los promedios finales obtenidos en sus respectivos cursos y su nivel de procrastinación académica mostrado en este estudio.

\section{RESULTADOS}

Los resultados de este estudio revelaron que $43(58,11 \%)$ de 74 participantes eran mujeres y 31 (41,89\%) de ellos eran hombres. En el primer constructo (elaboración de un trabajo final de curso), los resultados mostraron que el $75,4 \%$ de los estudiantes se encontraban en el nivel de procrastinación de moderado a alto; de ellos, el 26,6\% había practicado casi siempre o siempre la procrastinación. Aproximadamente el 65,7\% de ellos eran procrastinadores en un nivel moderado a alto al estudiar para un examen, entre los cuales el $30 \%$ eran académicamente siempre o casi siempre procrastinadores. 
Tabla 1

Procrastinación académica de estudiantes

\begin{tabular}{|c|c|c|c|c|c|c|}
\hline \multirow[b]{2}{*}{ Constructos / criterios } & \multicolumn{6}{|c|}{ Escala } \\
\hline & Nunca & $\begin{array}{c}\text { Casi } \\
\text { nunca }\end{array}$ & A veces & $\begin{array}{c}\text { Casi } \\
\text { siempre }\end{array}$ & Siempre & $\Sigma$ \\
\hline 1. Elaboración del trabajo final de & 11 & 7 & 36 & 15 & 5 & 74 \\
\hline curso & $14,86 \%$ & $9,46 \%$ & $48,65 \%$ & $20,27 \%$ & $6,76 \%$ & $100 \%$ \\
\hline 2. Estudiar para los exámenes & 18 & 5 & 27 & 20 & 4 & 74 \\
\hline & $24,32 \%$ & $6,76 \%$ & $36,49 \%$ & $27,03 \%$ & $5,41 \%$ & $100 \%$ \\
\hline 3. Cumplimiento de la asignación de & 19 & 4 & 34 & 16 & 1 & 74 \\
\hline lecturas semanales & $25,68 \%$ & $5,41 \%$ & $45,95 \%$ & $21,62 \%$ & $1,35 \%$ & $100 \%$ \\
\hline 4. Ejecución de actividades & 24 & 5 & 25 & 17 & 3 & 74 \\
\hline administrativas aca démicas & $32,43 \%$ & $6,76 \%$ & $33,78 \%$ & $22,97 \%$ & $4,05 \%$ & $100 \%$ \\
\hline 5. Cumplimiento de acciones de & 18 & 5 & 31 & 18 & 2 & 74 \\
\hline asistencia a reuniones & $24,32 \%$ & $6,76 \%$ & $41,89 \%$ & $24,32 \%$ & $2,70 \%$ & $100 \%$ \\
\hline 6. Ejecución de activida des aca démicas & 13 & 2 & 27 & 30 & 2 & 74 \\
\hline en general & $17,57 \%$ & $2,70 \%$ & $36,49 \%$ & $40,54 \%$ & $2,70 \%$ & $100 \%$ \\
\hline
\end{tabular}

En la primera dimensión de la procrastinación académica: elaboración de un trabajo final de curso, los resultados muestran que el $75,68 \%$ de los estudiantes se encuentran en el nivel de procrastinación de moderado a alto; de ellos, el $27,03 \%$ ha practicado casi siempre o siempre la procrastinación. Aproximadamente el $68,92 \%$ de ellos son procrastinadores en un nivel moderado a alto al estudiar para un examen, entre los cuales el $32,43 \%$ son académicamente siempre o casi siempre procrastinadores.

En cuanto a mantenerse al día con las asignaciones de lectura semanales, los resultados muestran que el $68,92 \%$ de los estudiantes bajo el estudio han practicado un nivel de procrastinación de moderado a alto, y el $22,97 \%$ siempre o casi siempre procrastinaban en el contexto académico. Aproximadamente el $60,81 \%$ son procrastinadores en un nivel de moderado a alto en la realización de tareas administrativas; entre ellos, el 27,03\% siempre o casi siempre muestra procrastinación académica. Además, se demuestra que el $68,92 \%$ de los estudiantes eran moderados o procrastinadores altos en la asistencia a las reuniones, de los cuales el $27,03 \%$ siempre o casi siempre procrastinadores. Finalmente, los hallazgos de este estudio revelan que el $79,73 \%$ de ellos tiene un nivel de dilación moderado a alto en la realización de tareas académicas en general, de los cuales el 43,24\% siempre o casi siempre son procrastinadores académicos (Tabla 1). En suma, los resultados evidencian que, de modo general, el $29,96 \%$ de los estudiantes siempre o casi siempre muestran un alto nivel de procrastinación.

\section{Tabla 2}

Rendimiento académico por conglomerados de los estudiantes

\begin{tabular}{|c|c|c|c|c|c|c|c|}
\hline \multirow{3}{*}{ Cursos } & & \multirow{3}{*}{$\mathrm{N}$} & \multicolumn{3}{|c|}{ Situación del estudiante al término del } & \multirow{3}{*}{ Media } & \multirow{3}{*}{$\begin{array}{l}\text { Desviación } \\
\text { estándar }\end{array}$} \\
\hline & & & & curso & & & \\
\hline & & & $\begin{array}{c}\text { Aprobó el } \\
\text { curso }\end{array}$ & $\begin{array}{c}\text { Desaprobó el } \\
\text { curso }\end{array}$ & $\begin{array}{l}\text { Se retiró } \\
\text { del curso }\end{array}$ & & \\
\hline $\begin{array}{l}\text { Lenguaje } \\
\text { comunicación }\end{array}$ & $\mathrm{y}$ & 21 & $\begin{array}{c}15 \\
71,43 \%\end{array}$ & $\begin{array}{c}3 \\
14,29 \%\end{array}$ & $\begin{array}{c}3 \\
14,29 \%\end{array}$ & 4,14 & 0,70 \\
\hline Redacción técnica & & 20 & $\begin{array}{c}17 \\
85,00 \%\end{array}$ & $\begin{array}{c}1 \\
5,00 \%\end{array}$ & $\begin{array}{c}2 \\
10,00 \%\end{array}$ & 4,50 & 0,50 \\
\hline Comprensión textual & & 33 & $\begin{array}{c}20 \\
60,61 \%\end{array}$ & $\begin{array}{c}6 \\
18,18 \%\end{array}$ & $\begin{array}{c}7 \\
21,21 \%\end{array}$ & 3,79 & 0,78 \\
\hline
\end{tabular}


En la Tabla 2 se muestra la situación final de los universitarios en sus respectivos cursos, lo cual demuestra la distribución del éxito académico por grupos. Asimismo, al contrastar con los resultados de la encuesta, en promedio el $27,66 \%$ de los estudiantes de los tres cursos (conglomerados) que desaprobaron o se retiraron del curso son los que mostraron los más altos niveles de procrastinación académica. Todo lo cual demuestra la hipótesis de que los estudiantes que procrastinan más son los que menor calificación final obtienen en sus cursos.

Tabla 3

Correlación entre procrastinación académica y rendimiento académico de estudiantes

\begin{tabular}{|c|c|c|c|c|c|c|}
\hline \multirow[b]{2}{*}{$\begin{array}{l}\text { Rendimiento } \\
\text { académico }\end{array}$} & \multicolumn{6}{|c|}{ Constructos de la procrastina ción académica de estudiantes } \\
\hline & $\begin{array}{c}\text { Elaboración } \\
\text { del trabajo } \\
\text { final de } \\
\text { curso }\end{array}$ & $\begin{array}{l}\text { Estudiar } \\
\text { para los } \\
\text { exámenes }\end{array}$ & $\begin{array}{l}\text { Cumplimiento } \\
\text { de la } \\
\text { asignación de } \\
\text { lecturas } \\
\text { semanales }\end{array}$ & $\begin{array}{l}\text { Ejecución de } \\
\text { actividades } \\
\text { administrativas } \\
\text { académicas }\end{array}$ & $\begin{array}{l}\text { Cumplimiento } \\
\text { de acciones } \\
\text { de asistencia } \\
\text { a reuniones }\end{array}$ & $\begin{array}{c}\text { Ejecución } \\
\text { de } \\
\text { actividades } \\
\text { académicas } \\
\text { en general }\end{array}$ \\
\hline $\begin{array}{c}\text { Coeficiente } \\
\text { de } \\
\text { correlación } \\
\text { de Pearson }\end{array}$ & $-0,30^{a}$ & $-0,27^{a}$ & $-0,28^{a}$ & $-0,30^{a}$ & $-0,21^{a}$ & $-0,34^{a}$ \\
\hline$p$ - valor & 0,01 & 0,01 & 0,01 & 0,01 & 0,01 & 0,01 \\
\hline
\end{tabular}

Según los resultados, el $48,65 \%$ creía que el hecho de postergar las tareas y actividades académicas les había resultado problemática. Por su parte, como se muestra en la Tabla 3 los resultados del análisis correlacional entre procrastinación académica y rendimiento académico, evidencian la existencia de una relación entre las dos variables. Se observa la capacidad predictiva del cuestionario de procrastinación académica respecto del rendimiento académico de los estudiantes universitarios. De esta forma, se establece una relación inversa significativa, lo cual permite comprender que los estudiantes que presentan calificaciones más altas en el curso respectivo muestran, por lo general, un menor nivel de dilación académica. En consecuencia, los estudiantes más procrastinadores muestran menor rendimiento académico e incluso se retiran del curso.

Tabla 4

Contraste de la procrastinación académica entre estudiantes de género masculino y femenino

\begin{tabular}{|c|c|c|c|}
\hline Procrastinación académica & Media \pm Desviación estándar & Prueba t-student & $p$-valor \\
\hline Género & & 3,195 & 0,01 \\
\hline Masculino & $3,10 \pm 0,88$ & & \\
\hline Femenino & $2,60 \pm 0,93$ & & \\
\hline
\end{tabular}

Según los resultados, se observa una diferencia significativa entre la procrastinación académica de los estudiantes hombres y mujeres, de modo que los estudiantes de género masculino muestran ser más procrastinadores que los estudiantes de género femenino (Tabla 3). Esto se refuerza más aún si identificamos por género a los estudiantes desaprobados o retirados, pues a pesar que en la muestra poblacional estudiada existe una mayor proporción de estudiantes de género femenino, los estudiantes desaprobados y los que se retiran son en su mayoría varones. 
Tabla 5

Principales causas de procrastinación académica

\begin{tabular}{|c|c|c|c|c|c|}
\hline \multirow[b]{2}{*}{ Constructos / criterios } & \multicolumn{3}{|c|}{ Escala } & \multirow[b]{2}{*}{ Media } & \multirow[b]{2}{*}{$\begin{array}{l}\text { Desviación } \\
\text { estándar }\end{array}$} \\
\hline & $\begin{array}{l}\text { No, de ningún } \\
\text { modo refleja } \\
\text { por qué yo } \\
\text { postergo la } \\
\text { tarea } \\
\end{array}$ & $\begin{array}{l}\text { Lo refleja en } \\
\text { cierto modo }\end{array}$ & $\begin{array}{c}\text { Sí, } \\
\text { definitivamente } \\
\text { refleja por qué } \\
\text { yo postergo la } \\
\text { tarea }\end{array}$ & & \\
\hline 1. Ansiedad por la evaluación & $\begin{array}{c}23 \\
31,08 \%\end{array}$ & $\begin{array}{c}9 \\
12,16 \%\end{array}$ & $\begin{array}{c}42 \\
56,76 \%\end{array}$ & 3,51 & 0,72 \\
\hline 2. Perfeccionismo & $\begin{array}{c}25 \\
33,78 \%\end{array}$ & $\begin{array}{c}22 \\
29,73 \%\end{array}$ & $\begin{array}{c}27 \\
36,49 \%\end{array}$ & 3,05 & 0,99 \\
\hline $\begin{array}{l}\text { 3. Dificultad para tomar } \\
\text { decisiones }\end{array}$ & $\begin{array}{c}5 \\
6,76 \%\end{array}$ & $\begin{array}{c}27 \\
36,49 \%\end{array}$ & $\begin{array}{c}42 \\
56,76 \%\end{array}$ & 4,00 & 0,67 \\
\hline $\begin{array}{l}\text { 4. Dependencia y búsqueda de } \\
\text { ayuda }\end{array}$ & $\begin{array}{c}18 \\
24,32 \%\end{array}$ & $\begin{array}{c}6 \\
8,11 \%\end{array}$ & $\begin{array}{c}50 \\
67,57 \%\end{array}$ & 3,86 & 0,56 \\
\hline $\begin{array}{l}\text { 5. Aversión a la tarea y baja } \\
\text { tolerancia a la frustración }\end{array}$ & $\begin{array}{c}2 \\
2,70 \%\end{array}$ & $\begin{array}{c}23 \\
31,08 \%\end{array}$ & $\begin{array}{c}49 \\
66,22 \%\end{array}$ & 4,27 & 0,95 \\
\hline 6. Falta de confianza en símismo & $\begin{array}{c}8 \\
10,81 \%\end{array}$ & $\begin{array}{c}29 \\
39,19 \%\end{array}$ & $\begin{array}{c}37 \\
50,00 \%\end{array}$ & 3,78 & 0,71 \\
\hline 7. Falta de aprobación & $\begin{array}{c}48 \\
64,86 \%\end{array}$ & $\begin{array}{c}1 \\
1,35 \%\end{array}$ & $\begin{array}{c}25 \\
33,78 \%\end{array}$ & 3,62 & 0,49 \\
\hline 8. Pereza & $\begin{array}{c}34 \\
45,95 \%\end{array}$ & $\begin{array}{c}14 \\
18,92 \%\end{array}$ & $\begin{array}{c}26 \\
35,14 \%\end{array}$ & 2,78 & 0,83 \\
\hline 9. Miedo al fracaso & $\begin{array}{c}1 \\
1,35 \%\end{array}$ & $\begin{array}{c}12 \\
16,22 \%\end{array}$ & $\begin{array}{c}61 \\
82,43 \%\end{array}$ & 4,62 & 0,93 \\
\hline $\begin{array}{l}\text { 10. Tendencia a sentirse } \\
\text { abrumado y administrar mal el } \\
\text { tiempo }\end{array}$ & $\begin{array}{c}15 \\
20,27 \%\end{array}$ & $\begin{array}{c}13 \\
17,57 \%\end{array}$ & $\begin{array}{c}46 \\
62,16 \%\end{array}$ & 3,84 & 0,72 \\
\hline 11. Rebelión contra el control & $\begin{array}{c}25 \\
33,78 \%\end{array}$ & $\begin{array}{c}39 \\
52,70 \%\end{array}$ & $\begin{array}{c}10 \\
13,51 \%\end{array}$ & 2,59 & 0,68 \\
\hline 12. Tomar riesgos & $\begin{array}{c}1 \\
1,35 \%\end{array}$ & $\begin{array}{c}26 \\
35,14 \%\end{array}$ & $\begin{array}{c}47 \\
63,51 \%\end{array}$ & 4,24 & 0,94 \\
\hline 13. Influencia de los compañeros & $\begin{array}{c}27 \\
36,49 \%\end{array}$ & $\begin{array}{c}13 \\
17,57 \%\end{array}$ & $\begin{array}{c}34 \\
45,95 \%\end{array}$ & 3,19 & 0,83 \\
\hline
\end{tabular}

Nivel del indicador: Muy bajo=1,00-1,49; Bajo=1,50-2,49; Medio =2,50-3,49; Al to = 3,50-4,49; Muy al to = 4,505,00

Los resultados de la Tabla 5 muestran las diferentes causas para procrastinar de los estudiantes en el plano académico. El constructo 9 es el criterio mejor calificado con una puntuación media muy alta de 4,62, lo que indica que la mayoría de los encuestados $(82,43$ $\%)$ están totalmente de acuerdo en que el miedo al fracaso definitivamente refleja el motivo por qué se posterga la tarea. El siguiente puntaje alto es el constructo 5 , con una puntuación media alta de 4,27 , lo que indica que la mayoría de los estudiantes $(66,22 \%)$ están totalmente de acuerdo en que la aversión a la tarea y la baja tolerancia a la frustración definitivamente reflejan el motivo por qué se posterga la tarea. Otro puntaje alto es el constructo 12 , con una puntuación media alta de 4,24, lo que indica que la mayoría de los estudiantes $(63,51 \%)$ están totalmente de acuerdo en que el preferir no tomar riesgos definitivamente refleja el motivo por qué se posterga la tarea. 


\section{DISCUSIÓN}

En el presente estudio, primero se determinó los niveles de procrastinación académica entre los estudiantes universitarios. Así, según los resultados, en general el $29,96 \%$ de los estudiantes fueron siempre o casi siempre procrastinadores; es decir, los resultados evidencian que un alto porcentaje de los estudiantes tienden a retrasar sus tareas académicas. Además, los resultados mostraron que existía una relación negativa significativa entre la postergación académica y el rendimiento académico de los estudiantes universitarios. El resultado obtenido respalda el argumento de Steel \& Gröpel (2008) quienes refieren que la procrastinación es generalmente perjudicial y nunca útil. Por lo tanto, se puede argumentar que los comportamientos de procrastinación casi nunca terminan con éxito. En este sentido, la investigación reafirma los estudios previos realizados con diferentes muestras que determinan que el vínculo entre la procrastinación y el rendimiento académico es negativo (Balkis \& Duru, 2019; Goroshit, 2018; Natividad, 2014). Por lo tanto, es probable que la procrastinación académica de los universitarios provoque escaso éxito y bajo desempeño académico, ya que en caso de que un estudiante no pueda tener éxito en la redacción de trabajos encargados finales o no estudie con el tiempo suficiente para el examen, obtendrá puntuaciones bajas.

En segundo lugar, los resultados en relación a las causas de la procrastinación académica y el rendimiento académico permiten identificar el papel de intermediación de otras variables académicas y motivacionales. Los resultados del estudio muestran que los aspectos motivacionales de las actividades educativas de los estudiantes universitarios, en el caso de la Universidad Nacional Agraria La Molina, se constituyen en una de las causas principales de la procrastinación académica. En tal sentido, cabe señalar que la insuficiencia motivacional se expresa en la procrastinación, la ansiedad y el deseo de los estudiantes por lograr resultados más externos de la actividad educativa en forma de obtención de una calificación (promedio ponderado), y no a aspectos internos centrados en el contenido del dominio significativo de los cursos (Karatas, 2015; De Palo, Monacis \& Sinatra, 2019; Manzanares, 2018). De este modo, se observa que el miedo al fracaso, la aversión a la tarea, baja tolerancia a la frustración, el tomar riesgos son los principales factores motivacionales que originan la procrastinación académica en los universitarios (Natividad, 2014; Wieland et al., 2021). Esta última causa (el tomar riesgos) demuestra que un grupo importante de estudiantes que procrastinan las actividades académicas les gusta trabajar bajo la presión del tiempo, a pesar de que ello le pueda generar estrés y afectar negativamente el desempeño académico.

Frente a todo lo anterior, una alternativa a esta situación es posibilitar formas separadas de apoyo motivacional para los estudiantes en la forma de apoyo psicológico individual y grupal de parte de la Oficina de Apoyo Psicológico del departamento médico de la universidad (Dikmen \& Bahadır, 2021). Asimismo, se puede inferir de los hallazgos que la procrastinación académica estudiantil no solo afecta al propio universitario, que retrasa la realización de la actividad o la entrega del trabajo, sino también a quienes deberían aceptar dicho trabajo, muchas veces cuando el tiempo ya se ha agotado; esto es, a los docentes. Por ello, los maestros tienen que dedicar su tiempo libre a ayudar a los estudiantes que postergan las cosas. Y como resultado de este fenómeno, los niveles de estrés de los profesores también pueden aumentar. 
La capacidad predictiva del cuestionario respecto de los niveles de rendimiento académico fue significativa ya que la prevalencia de la procrastinación académica refleja una tendencia de forma relativa y similar a los bajos niveles de desempeño académico o abandono de los cursos. En este sentido, algunos investigadores creen que el bajo nivel de desempeño al postergar a los estudiantes es el resultado de su baja autoeficacia (Steel \& Gröpel, 2008). Los estudiantes con bajos niveles de autoeficacia probablemente tengan miedo de aceptar y hacer las asignaciones, eviten trabajar, pospongan y abandonen pronto (Rahimi \& Vallerand, 2021). Además, otra razón del bajo logro de los individuos procrastinadores es, como lo demuestran algunos estudios, que estas personas poseen bajos niveles de estrategias de aprendizaje autorregulado y metacognitivo (Zacks \& Hen, 2018).

Algunos investigadores han definido la procrastinación como un problema en el desempeño de la autorregulación, lo cual conduce a la incapacidad para realizar o terminar sus asignaciones y tareas académicas. En consecuencia, los investigadores creen que las estrategias de aprendizaje autorregulado tienen un papel facilitador en el proceso de aprendizaje y los estudiantes con una variedad de estas estrategias aprenden más y se desempeñan mejor que sus compañeros que no son hábiles en el uso de estas estrategias (Manzanares, 2018; Visser, Korthagen \& Schoonenboom, 2018). También se encontró que había una diferencia significativa en la procrastinación entre estudiantes masculinos y estudiantes femeninos; los estudiantes varones practicaron más la procrastinación que sus condiscípulas.

En este sentido, los hallazgos en este estudio demuestran que las estudiantes actúan de forma más competitiva que los hombres en contextos académicos y están más motivadas para obtener calificaciones más altas; por lo tanto, tienen menos procrastinación académica. Las estudiantes son mejores que los hombres en estrategias de aprendizaje autorregulado y autoeficacia (Steel \& Gröpel, 2008). Además, los resultados han mostrado una relación negativa entre la autorregulación estrategias y procrastinación académica; los estudiantes que usan estas estrategias practican menos dilación en sus tareas académicas.

Por tanto, la dilación académica puede abordarse atendiendo la estrategia de Melih Dikmen \& Ferdi Bahadır (2021) quienes plantean la aplicación a los estudiantes procrastinadores de un tipo de orientación basada en el "enfoque de metas de aprendizaje"; con el propósito de lograr que los estudiantes sean más ambiciosos para adquirir conocimientos y empiecen a creer que pueden lograr sus objetivos. En cuanto a las prácticas pedagógicas con el fin de que los educandos eviten la dilación, se pueden establecer metas realistas y simples, con el fin de ayudar a disminuir los impactos negativos de la procrastinación. Por ello, es necesario que el estudiante se establezca plazos, aplique el tiempo eficientemente, utilice técnicas de gestión, divida las tareas en ciertos pasos pequeños y manejables, dándose recompensas convenientes, así como disminuya o elimine las distracciones (Loayza Maturrano, 2021). Todo lo cual puede ayudar a reducir la procrastinación.

Finalmente, en la presente investigación se emplea un cuestionario de percepción dirigido a los estudiantes, el cual puede haber supuesto algún sesgo. Además, en vista que los datos se recopilaron de los estudiantes universitarios de una sola institución educativa de nivel superior; esto podría limitar la generalización de los resultados a los estudiantes de otras universidades y otras carreras; más aún, si este fue un estudio de investigación transversal en el que los datos se recopilaron en un período de tiempo específico. Por lo tanto, se sugiere 
que se realicen más investigaciones de manera longitudinal para determinar el nivel de las conductas de procrastinación entre los estudiantes. Sin embargo, a pesar de las limitaciones mencionadas, la investigación actual se justifica y es relevante porque aumenta el conocimiento sobre el tema estudiado en los contextos universitarios.

\section{CONCLUSIONES}

En primer lugar, en términos generales, una conclusión principal es que los resultados revelaron que un número considerable de estudiantes practican la procrastinación académica en la mayoría de criterios. En particular, aquellos que practicaron más procrastinación tuvieron un rendimiento académico más pobre; por lo que se sugiere tomar medidas de prevención y remediación en los claustros universitarios. Todo lo cual corrobora la hipótesis general de investigación acerca de que la escala de procrastinación académica adaptada de Solomon y Rothblum (1984) permite predecir de forma significativa una correlación inversa respecto del nivel de rendimiento académico de los estudiantes universitarios.

Una segunda conclusión es que la procrastinación académica, que se manifiesta por el aplazamiento indefinido del desarrollo de las tareas educativas, es uno de los graves problemas académicos en el contexto universitario. En este estudio se demostró que los efectos de la procrastinación académica están asociados con el aumento del estrés, la ansiedad, el miedo, la culpa o vergüenza; es decir, vinculados a sentimientos de malestar interno. Estas causas fueron halladas en este estudio a través del cuestionario de procrastinación académica aplicado.

Asimismo, de la investigación se concluye que, con una alta probabilidad, el impacto de la dilación académica afecta no solo la vida académica del estudiante, sino también otras áreas de la vida en general. Consecuentemente, el estudiante universitario no puede dedicar su tiempo libre a lo que le agrada, porque está atormentado por el miedo a una tarea académica inconclusa, que está posponiendo. Todo lo cual provoca que el estudiante no tenga suficiente descanso para soportar con tranquilidad la carga académica necesaria calculada por la universidad, lo que significa que el estrés y la ansiedad no disminuyen en absoluto durante el semestre académico.

\section{REFERENCIAS}

Balkis, M. (2013). Academic procrastination, academic life satisfaction and academic achievement: The mediation role of rational beliefs about studying. Journal of EvidenceBased Psychotherapies, 13(1), 57-74. http://jebp.psychotherapy.ro/category/vol-xiii-no1-2013/

Balkis, M. \& Duru, E. (2019). Procrastination and Rational/Irrational Beliefs: A Moderated Mediation Model. Journal of Rational - Emotive and Cognitive - Behavior Therapy, 37(3), 299-315. https://doi.org/10.1007/s10942-019-00314-6

De Palo, V., Monacis, L., Miceli, S., Sinatra, M. \& Di Nuovo, S. (2017). Decisional procrastination in academic settings: The role of metacognitions and learning strategies. Frontiers in Psychology, 8(973). https://doi.org/10.3389/fpsyg.2017.00973 
De Palo, V., Monacis, L. \& Sinatra, M. (2019). How self-regulated learning strategies interfere between metacognitions and decisional procrastination. Psychology, Society and Education, 11(1), 39-52. https://doi.org/10.25115/psye.v11i1.1932

Díaz-Morales, J. (2019). Procrastinación: una revisión de su medida y sus correlatos. Revista Iberoamericana de Diagnóstico y Evaluación - e Avaliação Psicológica, 51(2), 43-60. https://doi.org/10.21865/RIDEP51.2.04

Dikmen, M. \& Bahadır, F. (2021). Goal Orientations as A Mediating Variable Between Academic Procrastination and Academic Achievement, International Journal of Eurasian Education and Culture, 6(13), 1028-1060. http://dx.doi.org/10.35826/ijoecc.404

Erazo, O. (2011). El rendimiento académico, un fenómeno de múltiples relaciones y complejidades. Revista vanguardia psicológica clínica teórica y práctica, 2(2), 144-173. https://dialnet.unirioja.es/servlet/articulo?codigo=4815141

Gómez, D., Martínez, E. y Oviedo, R. (2011). Factores que influyen en el rendimiento académico del estudiante universitario. Tecnociencia Chihuahua, 5(2), 90-97. https://vocero.uach.mx/index.php/tecnociencia/article/view/699

González-Pienda, J., Núñez, J., Álvarez, L., Roces, C., González-Pumariega, S., González, P., Muñiz, R., Valle, A., Cabanach, R., Rodríguez, S y Bernardo, A. (2003). Adaptabilidad y cohesión familiar, implicación parental en conductas autorregulatorias, autoconcepto del estudiantes y rendimiento académico. Psicothema, 15(3), 471-477. http://www.psicothema.com/pdf/1090.pdf

Goroshit, M. (2018). Academic procrastination and academic performance: An initial basis for intervention. Journal of Prevention \& Intervention in the Community, 46(2), 131-142. https://doi.org/10.1080/10852352.2016.1198157

Grunschel, C., Schwinger, M., Steinmayr, R. \& Fries, S. (2016). Effects of using motivational regulation strategies on students' academic procrastination, academic performance, and well-being. Learning and Individual Difference, 49, 162-170. https://doi.org/10.1016/j.lindif.2016.06.008

Hen, M. \& Goroshit, M. (2020). The effects of decisional and academic procrastination on students' feelings toward academic procrastination. Current Psychology, 39, 556-563. https://doi.org/10.1007/s12144-017-9777-3

Hernández, G., Paredes, V. y Martín, M. (2021). Factores que influyen en el rendimiento académico de los estudiantes de nivel superior en Tlaxcala derivado de la educación virtual durante la pandemia 2020. Brazilian Journal of Business, 3(2). https://doi.org/10.34140/bjbv3n2-011

Karatas, H. (2015). Correlation among academic procrastination, personality traits, and academic achievement. Anthropologist, $20 \quad(1, \quad 2), \quad 243-255$. https://doi.org/10.31901/24566802.2015/20.1-2.27

Krispenz, A., Gort, C., Schültke, L. \& Dickhäuser, O. (2019). How to reduce test anxiety and academic procrastination through inquiry of cognitive appraisals: A pilot study investigating the role of academic self-efficacy. Frontiers in Psychology, 10(1917). https://doi.org/10.3389/fpsyg.2019.01917

Loayza, E. (2006). Investigación cualitativa en Educación. Investigación educativa, 10(18), 75 85. https://revistasinvestigacion.unmsm.edu.pe/index.php/educa/article/view/3778

Loayza Maturrano, E. (2020). La investigación cualitativa en Ciencias Humanas y Educación. Criterios para elaborar artículos científicos. Educare et Comunicare, 8(2), 56-66. https://doi.org/10.35383/educare.v8i2.536 
Loayza Maturrano, E. (2021). El fichaje de investigación como estrategia para la formación de competencias investigativas. Educare et Comunicare, 9(1), 67-77. https://doi.org/10.35383/educare.v9i1.594

Manzanares, E. (2018). Procrastinación, creencias irracionales/racionales académicas y rendimiento académico en estudiantes de Beca 18 de una institución privada de Lima. (Tesis de maestría). Facultad de Ciencias de la Comunicación, Turismo y Psicología. https://repositorio.usmp.edu.pe/handle/20.500.12727/3546

Marquina, R., Gómez, L., Salas, C., Santibañez, S. y Rumiche, R. (2016). Procrastinación en alumnos universitarios de Lima Metropolitana. Revista Peruana de Obstetricia y $\begin{array}{lll}\text { Enfermería, 12(1), 11-22. } & \end{array}$ https://www.aulavirtualus mp.pe/ojs/index.php/rpoe/a rticle/view/747

Medina, N., Ferreira, J., y Marzo, M. (2018). Factores personales que inciden en el bajo rendimiento académico de los estudiantes de geometría. Revista Telos, 20, 14-28. http://ojs.urbe.edu/index.php/telos/article/view/899/846

Namakforoosh, M. (2009). Metodología de la Investigación. 2da. Ed. Editorial Limusa. Natividad, L. (2014). Análisis de la procrastinación en estudiantes universitarios. (Tesis doctoral) Facultad de Psicología de la Universidad de Valencia. https://roderic.uv.es/handle/10550/37168

Pérez-Mato, D. y Sierra-Salcedo, R. (2010). Diagnóstico, prevención y tratamiento de estudiantes universitarios con bajo rendimiento académico. Varona, (50), 29-36. https://www.redalyc.org/pdf/3606/360635568006.pdf

Quant, D. y Sánchez, A. (2012). Procrastinación, procrastinación académica: concepto e implicaciones. Revista vanguardia psicológica clínica teórica y práctica, 3(1), 45-59. https://dialnet.unirioja.es/servlet/articulo?codigo=4815146

Rahimi, S., \& Vallerand, R. (2021). The role of passion and emotions in academic procrastination during a pandemic (COVID-19). Personality and Individual Differences, 179, 110852. https://doi.org/10.1016/j.paid.2021.110852

Schouwenburg, H. (2004). Procrastination in Academic Settings: General Introduction. In H. Schouwenburg, C. Lay, T. Pychyl, \& J. Ferrari (Eds.), Counseling the procrastinator in academic settings, 3-17. American Psychological Association. https://doi.org/10.1037/10808-001

Steel, P. \& Gröpel, P. (2008). A mega-trial investigation of goal setting, interest enhancement, and energy on procrastination. Personality and Individual Differences, 45(5), 406-411. https://doi.org/10.1016/j.paid.2008.05.01.015

Valenzuela R., Codina N., Castillo I., Pestana J. (2020). Young university students' academic self-regulation profiles and their associated procrastination: Autonomous functioning requires self-regulated operations. Frontiers of Psychology, 11(354), 1-15. https://doi.org/10.3389/fpsyg.2020.00354

Vargas, M. y Montero, E. (2016). Factores que determinan el rendimiento en matemáticas en el contexto de una universidad tecnológica: aplicación de un modelo de ecuaciones $\begin{array}{llll}\text { estructurales. Universitas 15(4). } & \text { Psychológica, }\end{array}$ http://www.redalyc.org/jatsRepo/647/64748791025/64748791025.pdf

Visser, L., Korthagen, F. \& Schoonenboom, J. (2018). Differences in learning characteristics between students with high, average, and low levels of academic procrastination: Students 'views on factors influencing their learning. Frontiers of Psychology, 2018, 9(808). https://doi.org/10.3389/fpsyg.2018.00808 
Wieland, L., Ebner-Priemer, U., Limberger, M., \& Nett, U. (2021). Predicting delay in goaldirected action: an experience sampling approach uncovering within-person determinants involved in the onset of academic procrastination behavior. Frontiers in Psychology, 3078. https://doi.org/10.3389/fpsyg.2021.695927

Zacks, S. \& Hen, M. (2018). Academic interventions for academic procrastination: A review of the literature. Journal of Prevention \& Intervention in the Community, 46(2), 117-130. https://doi.org/10.1080/10852352.2016.1198154 